\title{
Problem solving in the rat: Septal lesion effects on habituation and perseverative tendencies
}

\author{
PAUL ELLEN and SUSAN L. WESTON \\ Georgia State University, Atlanta, Georgia
}

\begin{abstract}
The performance deficit that typically occurs on Maier's three-table spatial integration task following septal damage was examined with the view towards relating it to lesion-induced alterations in exploratory activity during the exploration phase of the task. Septals, unlike shams, failed to habituate (i.e., reduce the rate of sector entry behavior) during the 15-min exploration period. However, this failure to habituate was not the sole basis of the septal deficit on the spatial integration aspect of the task, since some animals that did habituate also failed to adopt the spatial solution. Unlike shams, septals tended to perseverate a turning response, not only on each of the three daily test trials, but also from day to day. This perseverative tendency was unresponsive to its consequences. Since the perseverative tendency did not correlate with any response bias expressed during the exploration phase of the task, it was regarded as the septal animal's adaptation to the test trial situation.
\end{abstract}

Successful problem solving on tasks involving cognitive mapping abilities (Ellen, Parko, Wages, Doherty, \& Herrmann, 1982) generally requires a prior exploration of the problem space (Stahl \& Ellen, 1974; Herrmann, Bahr, Bremner, \& Ellen, 1982). This requirement has been amply documented on the Maier three-table spatial integration task, in which animals are required to return to the baited table on a test trial after first having explored the entire unbaited complex of tables and runways and then having been given a short feeding experience on the baited table. Since the animals are started from and fed on tables that are varied daily, there is no possibility for the animals to learn either a constant locus of food or route to the food. Thus, each day the correct response reflects the integration of new information (daily locus of food), with the cognitive representation of the problem space acquired or reactivated during the exploratory period. Inasmuch as the information concerning the locus of food is changed on a daily basis, this integration of the feeding locus and the spatial relationships existing between the tables actually reflects a reorganization of memorial representations (Hermann et al., 1982).

Neurobehavioral studies by Hermann, Black, Anschel, and Ellen (1978), Hermann, Black, Doherty, and Ellen (1980), Rabe and Haddad (1969), and Stahl and Ellen $(1973,1979)$, have shown that lesions of

We would like to acknowledge the advice and contributions of C. Wages and Thom Herrmann in the design of the experiment and the preparation of this report. We also extend special thanks to James $\mathbf{L}$. Pate for assistance with the statistical analyses and Donna Floyd for competent secretarial assistance in the production of the various drafts of this paper. Our mailing address is: Department of Psychology, Georgia State University, Atlanta, Georgia 30303. the septal area and hippocampus and their interconnecting fiber systems markedly impair performance on the three-table task. Moreover, Hermann et al. (1980) have shown that the addition of an extramaze cue light to signal the locus of the baited table does not result in correct performance in septal and fornically damaged animals.

There is relatively little information available as to the basis of the deficit following septal, fornical, or hippocampal lesions. In examining this deficit, Stahl and Ellen $(1973,1979)$ found that septal rats tended to engage in more table entries during the 15-min exploratory phase of the problem than did normal animals, and, furthermore, the number of table entries for septals increased over days of testing. However, Stahl and Ellen did not distinguish between exploratory activity and general activity; thus, it is not clear from the Stahl and Ellen data whether the increased number of table entries shown by the septal animals reflected an increase in exploratory activity per se or merely an enhancement of general activity. That this distinction is not trivial is emphasized by the fact that O'Keefe and Nadel (1978) have also drawn attention to it in their development of the cognitive mapping theory of the hippocampus. O'Keefe and Nadel took the position that cognitive maps were acquired as an animal explored its environment and that the hippocampus provided the necessary anatomical substrate for exploratory behavior. Animals without a hippocampus presumably did not engage in exploratory behavior, although they might exhibit considerable locomotor activity. O'Keefe and Nadel operationalized the distinction between general activity and exploration by pointing out that general activity can only be measured under constant and familiar environmental 
conditions. Such activity is cyclical and shows marked increases in response to motivational or stimulatory conditions (food and water deprivation, for example). In contrast, exploratory activity generally decreases in response to motivational factors and is manifested primarily as a decrease in activity (habituation) within a novel environment as the animal becomes familiar with that environment.

From this perspective, it seems possible that the greater number of table entries by septal animals in the Stahl and Ellen studies $(1973,1979)$ reflected a higher level of general activity rather than a greater amount of exploratory behavior. Moreover, to the extent that the exploratory phase of the three-table task regularly preceded the feeding phase, it is entirely possible that table entry behavior itself could have acquired motivational significance for the animals in much the same way as other activities, such as running-wheel behavior or barpressing behavior on a fixed-interval operant schedule (Ellen \& Powell, 1962b), acquire motivational significance when they regularly precede feeding (Bolles, 1967). The increase in table-entry behavior of septal rats with repeated daily exposure to the apparatus is quite consistent with observations of barpressing behavior, which also tend to increase over days as this behavior becomes associated with feeding (Ellen \& Powell, 1962a).

The question is thus raised as to whether, indeed, the septal animals engage in exploratory behavior when they are actively locomoting during the exploratory phase of the three-table task. That exploration is extremely critical for performance on the threetable task has been documented by Stahl and Ellen (1974) and Herrmann et al. (1982). Normal animals not given an opportunity to explore prior to feeding fail to return to the baited table on the test trial. Moreover, merely allowing animals to run on limited parts of the apparatus (either tables only or runways only) does not result in successful performance on the three-table task (Ellen et al., 1982). Thus, running on the apparatus during test trials only or running on limited sections of the apparatus during the exploratory phase is not sufficient to ensure that the necessary cognitive representation will be acquired. Thus, exploration is different from mere locomotion or running from one place to another. It would seem to be an active information-gathering process, and, in the case of the three-table task, one that yields information concerning the spatial relationships existing among the tables. It follows from this analysis that, to the extent an animal can perform successfully on the three-table task, it has indeed engaged in exploration during the exploratory phase of the problem. The present experiment was concerned with determining whether the converse was true. Was the failure of septal-lesioned animals to perform correctly related to a failure to explore, despite enhanced activity during the exploratory phase?

\section{METHOD}

\section{Subjects}

Nineteen Long-Evans hooded male rats, approximately 180 days of age, were maintained at $85 \%-90 \%$ of their ad-lib body weights (i.e., the average weight on the 3 days prior to surgery during which days the animals had unlimited access to food). All animals were housed in individual cages. The animals had been handled extensively during a previous operant conditioning study and hence were not experimentally naive.

\section{Apparatus}

A variant of the Maier three-table apparatus (Maier, 1932b) was used in this experiment. It consisted of three pathways, $61 \mathrm{~cm}$ long and $7.5 \mathrm{~cm}$ wide, that were elevated $59 \mathrm{~cm}$ from the floor and joined at equal angles. The three tables, one of which was placed at the end of each runway, differed in the nature of their top surfaces and in the nature of the extramaze stimuli associated with them. A $63 \times 49 \mathrm{~cm}$ screen with a $10 \times 10 \mathrm{~cm}$ opening was attached to the front edge of each table in order to obstruct the view of the tables from other parts of the apparatus. The entire complex was painted flat black and located inside a $206 \times 174 \times 205 \mathrm{~cm}$ wire enclosure that was illuminated from above by standard fluorescent fixtures.

\section{Behavioral Procedures}

The animals received 5 days of handling and board training prior to surgery in an area outside of the experimental cubicle. In board training, an animal was placed on the center of a board suspended between two baited chairs and allowed to run back and forth between the chairs for $3 \mathrm{~min}$.

Four to 6 days postoperatively, testing on the three-table apparatus was initiated. This testing consisted of three phases. In the first phase, the animals were placed at the intersection of the runways in groups of four or five and allowed to run over the runways and tables for $15 \mathrm{~min}$. At this time, neither the tables nor the runways were baited. This period was designated as the exploratory phase of the problem. During this period, whenever an animal moved all four legs from one runway to another, it was given credit for a sector entry. The number of sector entries in each of the successive 3-min intervals of the 15-min exploratory period was recorded for each animal. This procedure differed from that used by Stahl and Ellen $(1973,1979)$ in two distinct ways. First, Stahl and Ellen recorded only the total number of table entries in the 15-min exploratory period, and second, they recorded a table entry whenever the animal crossed from a runway to a table. While the latter is perhaps less important relative to the method used in the present experiment, the failure of Stahl and Ellen to break down the total number of entries in the 15-min exploratory period into smaller time segments precluded the analysis in their work of table-entry behavior in terms of exploration within a daily session.

Following the 15-min exploration phase, the animals were removed from the apparatus and placed in a group restraining cage. The table that was to be that day's baited table was baited with a dish of wet mash, while one of the other tables was similarly baited. However, the food on the latter table was securely covered with a wire mesh, making it unavailable to the animal. A group of four or five animals was placed on the baited table and allowed to feed for $2 \mathrm{~min}$. The animals were not allowed to deplete the food supply during this activity. After this feeding phase, all rats were removed from the apparatus and returned to the group restraining cage. Each rat was individually removed from the restraining cage and placed on the start table for testing. Guillotine doors were raised on the baited table and the table containing the wireenclosed food. Then the guillotine door on the start table was raised and the animal was allowed 3 min to go from the start table to the baited table. If an animal did not move within $3 \mathrm{~min}$, or went to the table with the wire-enclosed food, an error was scored; if an animal left the baited table without feeding, the data for that 
trial was not included in data calculations. Half of the animals in both the sham-operated and septal groups were allowed to correct a commission error by leaving the incorrect table and making another choice. The remaining animals in each group were immediately removed from the incorrect table. Three such trials were given to each rat daily for 24 days. The start and baited tables were varied each day in a semirandom manner so that no start table or turn was repeated for more than 2 successive days, thereby preventing the learning of a locus or route to the food.

\section{Surgical Procedures}

The animals were assigned randomly to one of four groups, two of which were to receive septal lesions $(\mathrm{N}=10)$ and two of which were to be given sham operations $(\mathbf{N}=9)$. Sodium pentobarbital (Nembutal) for anesthesia $(40 \mathrm{mg} / \mathrm{kg}$ ) and $0.2 \mathrm{cc}$ atropine sulfate to reduce respiratory distress were injected intraperitoneally 30 min prior to surgery. Subsequently, the animals were placed in a Kopf stereotaxic device with the upper incisor bar set at $+5 \mathrm{~mm}$ above the horizontal zero plane. Lidocaine hydrochloride (Xylocaine) was infiltrated into the scalp as a local anesthetic prior to a midline incision's being made. Bregma was identified and a single burr hole was drilled $2 \mathrm{~mm}$ anterior to bregma. An insulated electrode with an exposed tip of $0.5 \mathrm{~mm}$ was lowered $5 \mathrm{~mm}$ below the surface of the brain on the midline. For $20 \mathrm{sec}$, $2 \mathrm{~mA}$ of current from a Stoelting electrolytic lesion-producing device (Model 58040) were passed between the anodal electrode in the brain and a cathodal clip attached to the wound margin. Following the passing of the current, the electrode was retracted and the wound was sutured with silk thread. Penicillin $(.2 \mathrm{cc})$ was injected intramuscularly prior to removal from the stereotaxic device. Animals given sham operations were treated in exactly the same manner, with the exception that current was not passed after the electrode had been lowered into the brain.

\section{Histological Procedures}

Upon completion of the experiment, all animals were sacrificed with a lethal dose of Nembutal $(0.9 \mathrm{cc})$. The animals were perfused intracardially with saline, followed by a $10 \%$ Formalin solution. The brains were removed and stored in Formalin. Frozen sections, $50 \mu \mathrm{m}$ thick, were prepared, with every fifth section mounted and stained with cresyl violet for examination of lesion locus and extent.

\section{RESULTS}

\section{Histological Findings}

Of the 10 lesions aimed at the septal area, three distinct groupings emerged (see Figure 1). The largest group $(\mathbf{N}=5)$ tended to be anterior, somewhat unilateral, and localized in the precommissural septal area. No lesion in this group extended posterior to the crossing of the anterior commissure. The next group consisted of three animals whose lesions were quite similar to those of the first group, with the exception that they tended to extend to the area immediately posterior to the anterior commissure. The third grouping consisted of two animals whose lesions extended from a plane anterior to the crossing of the anterior commissure and proceeded posteriorly to the hippocampal commissure, fornix, and nucleus triangularis septi. In view of the similarity in extent of damage and the fact that no structures other than those of the septal-hippocampal complex were implicated in the lesions, all lesions were considered as belonging to the population of septal le-

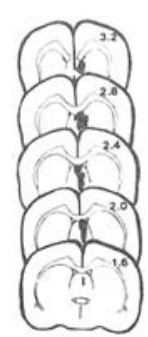

sc3

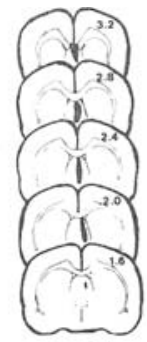

SC4

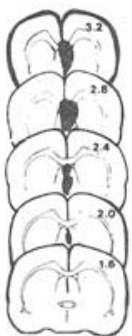

SNC1

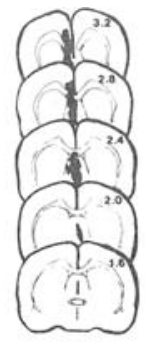

SC1

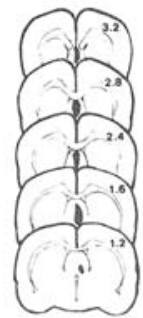

SNC2

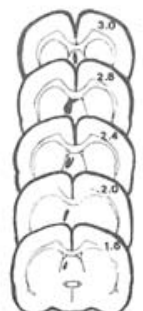

SC5
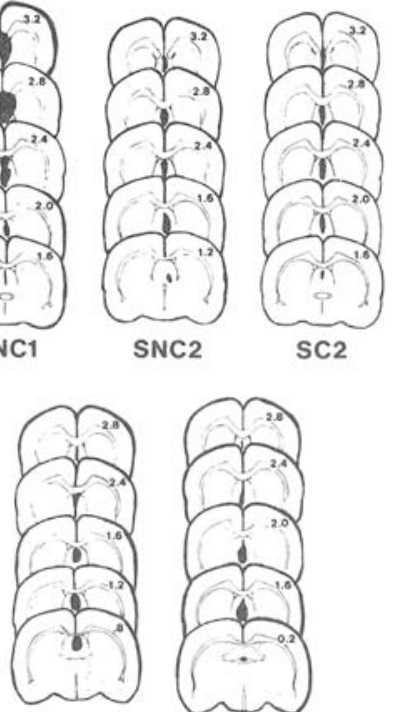

SNC5

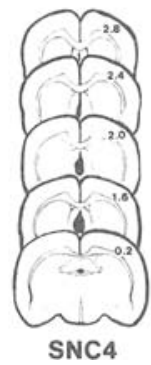

Figure 1. Reconstruction of septal lesions on plates of the Pellegrino and Cushman (1967) atlas.

sions and data from all animals were included for data analyses.

\section{Behavioral Findings}

Since the first 4 days of testing occurred within 10 days of surgery, and since none of the animals had had any preoperative adaptation to the threetable apparatus, data from these 4 days were eliminated (Maier, 1932a; Stahl \& Ellen, 1973) and only the data from Days 5-24 of testing were analyzed and included in the present report.

\section{Test Trial Behavior}

It will be recalled that the choice behavior on the first of the three daily test trials reflects the ability of animals to integrate the information concerning the varied daily locus of food with the constant spatial relations existing among the tables acquired during the daily exploratory experience. A score of twice as many correct as incorrect $(67 \%)$ is regarded as evidence of successful integrative performance, inasmuch as that level of performance or better occurs only $5 \%$ of the time by chance alone for the 20 test trials given. 
Table 1 shows the average performance for each animal in all four groups. The data for Trial 1 reveals that no septal animal scored above the criterion, and the group as a whole averaged $53 \%$ correct over the course of testing. In contrast, 5/9 of the sham-operated animals performed at or above criterion level. This difference in the number of animals meeting the criterion is significant $(p=.025$, Fisher's exact probability test). Thus, it is clear that animals sustaining septal damage are impaired in performance on the spatial integration trial of the three-table task.

Table 1 also shows no difference on Trial 1 performance between those animals allowed to correct an error on all test trials and those not allowed the correction opportunity. In the former group, shams averaged $74 \%$ correct, as compared with $52 \%$ correct for septals in this group. For those animals not given the correction opportunity, shams averaged $75 \%$ correct, while septals averaged $55 \%$ correct over the 20 days of testing. It is clear from these findings that the opportunity to correct the first choice on each test trial played no significant role in the Trial 1 performance.

Not only did the correction opportunity not facilitate Trial 1 performance, it also failed to have a beneficial effect on Trials 2 and 3. As can be seen from Table 1, the Trial 2 and Trial 3 performance of shams as well as septals, regardless of correction opportunity, was not materially better than their Trial 1 performance.

The next question of interest is whether septals or shams tended to repeat on the second test of a day the response made on the first trial (stay) or whether they would make a different response (shift) on the second test trial. For each day, eight outcomes were possible for each animal: two reflected a correct choice on Trials 1 and $2(+++,++-)$; two outcomes reflected an incorrect choice on Trials 1 and $2(---,--+)$. In each of these instances, however, there is a repetition of the preceding choice. Four outcomes reflected a choice on Trial 2 that was different from that on Trial $1(-++,-+-,+--$, $+-+)$. No statistically reliable difference in the relative frequency of either the stay or shift type of response pattern on Trial 2 was found between animals given the correction opportunity and those not given such an opportunity. This observation was true for both septals and shams. In view of the general ineffectiveness of the correction opportunity, the data from both correction and noncorrection groups were pooled and all subsequent analyses were carried out on the pooled data.

Since 19 animals were tested for 20 days, 380 stay and shift outcomes were possible. Of these 380 outcomes, there were 243 in which the animals were correct on the first trial and 137 in which the animals were incorrect on the first trial. Table 2 shows the distribution of stay and shift responses on the second daily test trial for both septals and shams following either a correct or an incorrect outcome on the first trial. As can be noted from the table, after a correct first-trial outcome, both groups tended to stay with that response on the second trial $\chi^{2}=2.65$, n.s.). In contrast, when the outcome was incorrect on the first trial, shams tended to shift responses for the second trial, whereas septals tended to repeat the incorrect response on the second trial $\left(\chi^{2}=26.4, p<\right.$

Table 1

Test Trial Performance on the Three-Table Task

\begin{tabular}{|c|c|c|c|c|c|c|c|}
\hline \multirow[b]{3}{*}{ Rat } & \multirow{2}{*}{\multicolumn{3}{|c|}{$\begin{array}{c}\text { Correction Opportunity } \\
\text { Test Trial } \\
\end{array}$}} & \multirow[b]{3}{*}{ Rat } & \multicolumn{3}{|c|}{ No Correction Opportunity } \\
\hline & & & & & \multicolumn{3}{|c|}{ Test Trial } \\
\hline & 1 & 2 & 3 & & 1 & 2 & 3 \\
\hline \multicolumn{8}{|c|}{ Sham } \\
\hline $\begin{array}{l}\text { ShC1 } \\
\text { ShC2 } \\
\text { ShC3 } \\
\text { ShC4 }\end{array}$ & $\begin{array}{l}50 \\
65 \\
90 \\
90\end{array}$ & $\begin{array}{l}50 \\
85 \\
72 \\
65\end{array}$ & $\begin{array}{l}55 \\
70 \\
59 \\
80\end{array}$ & $\begin{array}{l}\text { ShNC1 } \\
\text { ShNC2 } \\
\text { ShNC3 } \\
\text { ShNC4 } \\
\text { ShNC5 }\end{array}$ & $\begin{array}{l}75 \\
95 \\
60 \\
60 \\
85\end{array}$ & $\begin{array}{l}67 \\
90 \\
75 \\
70 \\
95\end{array}$ & $\begin{array}{l}60 \\
85 \\
65 \\
55 \\
90\end{array}$ \\
\hline $\begin{array}{l}\text { Mean } \\
\text { SD }\end{array}$ & $\begin{array}{r}74 \\
5.3\end{array}$ & $\begin{array}{r}68 \\
7.0\end{array}$ & $\begin{array}{r}66 \\
3.8\end{array}$ & $\begin{array}{l}\text { Mean } \\
\text { SD }\end{array}$ & $\begin{array}{r}75 \\
6.8\end{array}$ & $\begin{array}{r}79 \\
3.9\end{array}$ & $\begin{array}{r}71 \\
4.4\end{array}$ \\
\hline \multicolumn{8}{|c|}{ Septal } \\
\hline $\begin{array}{l}\text { SC1 } \\
\text { SC2 } \\
\text { SC3 } \\
\text { SC4 } \\
\text { SC5 }\end{array}$ & $\begin{array}{l}53 \\
45 \\
50 \\
50 \\
60\end{array}$ & $\begin{array}{l}67 \\
53 \\
70 \\
55 \\
70\end{array}$ & $\begin{array}{l}67 \\
47 \\
65 \\
50 \\
60\end{array}$ & $\begin{array}{l}\text { SNC1 } \\
\text { SNC2 } \\
\text { SNC3 } \\
\text { SNC4 } \\
\text { SNC5 }\end{array}$ & $\begin{array}{l}47 \\
47 \\
60 \\
65 \\
55\end{array}$ & $\begin{array}{l}53 \\
47 \\
70 \\
60 \\
50\end{array}$ & $\begin{array}{l}53 \\
58 \\
80 \\
50 \\
55\end{array}$ \\
\hline $\begin{array}{l}\text { Mean } \\
\text { SD }\end{array}$ & $\begin{array}{r}52 \\
2.9\end{array}$ & $\begin{array}{r}63 \\
1.9 \\
\end{array}$ & $\begin{array}{r}58 \\
3.0\end{array}$ & $\begin{array}{l}\text { Mean } \\
\text { SD }\end{array}$ & $\begin{array}{r}55 \\
3.5\end{array}$ & $\begin{array}{r}56 \\
4.0\end{array}$ & $\begin{array}{r}59 \\
6.9\end{array}$ \\
\hline
\end{tabular}

Note-Percent correct in 20 trials. 
Table 2

Daily Trial 2 Performance as a Function of Outcome of Trial 1

\begin{tabular}{lccrrr}
\hline & \multicolumn{3}{c}{ Trial 1 Outcome } \\
\cline { 2 - 5 } \multicolumn{1}{c}{ Trial 2 } & Stay (+) & Shift $(-)$ & Stay $(-)$ & Shift $(+)$ \\
\cline { 2 - 5 } \cline { 5 - 6 } Sham-Operatees & 98 & 40 & 7 & 35 \\
Septals & 85 & 20 & 65 & 30 \\
\hline
\end{tabular}

.001). It would appear that septals perseverate in a response despite the consequences of that response.

The tendency of septal animals to perseverate from trial to trial within a single day is also manifested over successive days of testing. In the present experiment, the particular random order of start tables and turns used would cause an animal to make the same turn only $55 \%$ of the time on Trial 1 if it were to make a perfect score over the 20 test days. Shams, regardless of whether they reached the criterion level for Trial 1 , made the same turn only $62 \%$ of the time. In contrast, septals actually turned in the same direction $82 \%$ of the time. Although it would appear that both septals and shams show a tendency to repeat the same turn on subsequent days, this tendency was not correlated with the tendency to repeat the same turn on Trials 2 and 3 of a single test day in the case of the shams $\left(r_{s}=-.2068, p>.20\right)$, but was in the case of septals $\left(r_{s}=+.7256, p<.01\right)$. Thus, it would appear that, for septal animals, there is a generalized tendency to repeat the same turning response both within and between days.

\section{Exploratory Behavior}

Daily activity during the exploratory phase of the problem in the last 20 days of the 24-day test period is shown in Figure 2. It will be recalled that, during the exploratory phase of the problem, animals were placed in the center of the apparatus and a score was recorded whenever an animal moved all four legs from one sector of the apparatus to another. The average number of sectors entered per minute of the 15-min exploratory period constituted the basic data for this figure. Operated animals had a higher rate of sector entries than did shams; however, despite daily fluctuations in the rate of sector entries, the overall rate for the septals was relatively constant over the 20 days. In contrast, the shams showed a steady increase in the rate of sector entries over the 20 days of testing. A repeated-measures ANOVA with days as the repeated-measure factor and lesion vs. sham as the between-groups factor yielded significant $F$ ratios for lesions $[F(1,17)=43.70, p<$ $.0001]$, days $[F(19,323)=2.58, p<.0004]$, and the days $\times$ lesion interaction $[F(19,323)=1.81, p=$ $.021]$, confirming the apparent differences in sectorentry rate over days. Trend analysis of the days $x$ lesion interaction revealed significant differences in the linear trend between the normals and septals $[\mathrm{F}(1,17)=4.91, \mathrm{p}<.04]$.

In addition to obtaining an overall measure of daily exploratory activity, changes in exploratory behavior within each day's exploratory phase were quantified. The 15-min exploratory phase was broken down into five successive 3-min epochs, and the sector entry behavior for each of these epochs was averaged over all days of testing. Figure 3 shows the rate of sector entries averaged over days for successive 3-min epochs of the 15-min exploratory phase. Septal animals show approximately the same rate of sector entries at the end of the 15-min exploratory period, as they did at the beginning of that period. In contrast, for shams, the rate of sector entries is high early in the exploratory period (first $3 \mathrm{~min}$ ) and declines during the remaining minutes of the exploratory phase. A repeated-measures ANOVA with successive intervals as the repeated-measure factor and lesions as the between-groups factor yielded a significant lesion $\times$ interval interaction $[F(4,68)$ $=5.25, \mathrm{p}<.001]$, as well as a significant main effect for interval $[F(4,68)=2.95, p=.03]$. Trend analysis of the lesion $x$ interval interaction indicated a significant difference in both the linear and quadratic components $[F(1,17)=6.86, p<.02$, and $F(1,17)$ $=6.55, \mathrm{p}=.02$, respectively]. It is thus clear that, while the shams demonstrate habituation (reduced rate of sector entries) during the 15-min exploratory phase, the septal animals, on the average, do not.

The question that motivated the present study was whether the failure of septal animals to perform successfully on Trial 1 could be related to an absence of exploratory activity as measured by habituation during the exploratory phase. For each animal, a habituation index was computed by determining the relative decrease in sector-entry rate from the first $3 \mathrm{~min}$ of the exploratory period (A) to the last $3 \mathrm{~min}$ (B) according to the formula $(A-B) / A$. The value

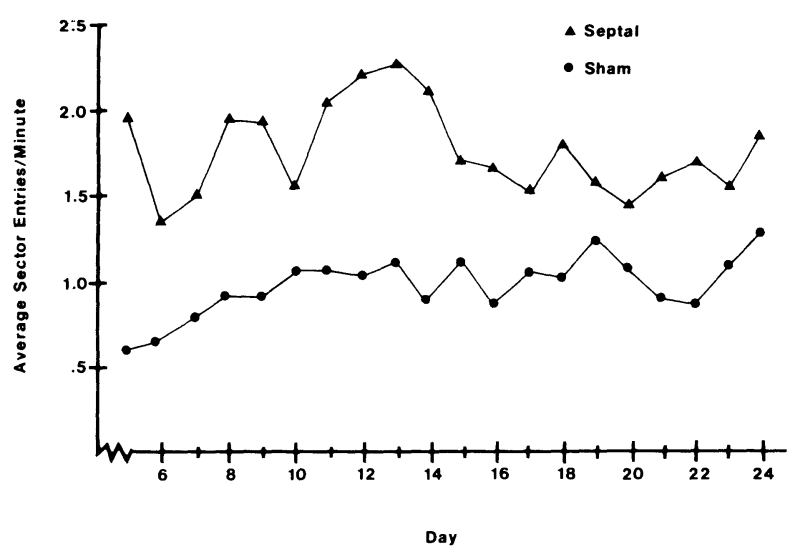

Figure 2. Rate of sector-entry behavior for septal and normal rats during the daily 15-min exploratory phase. 


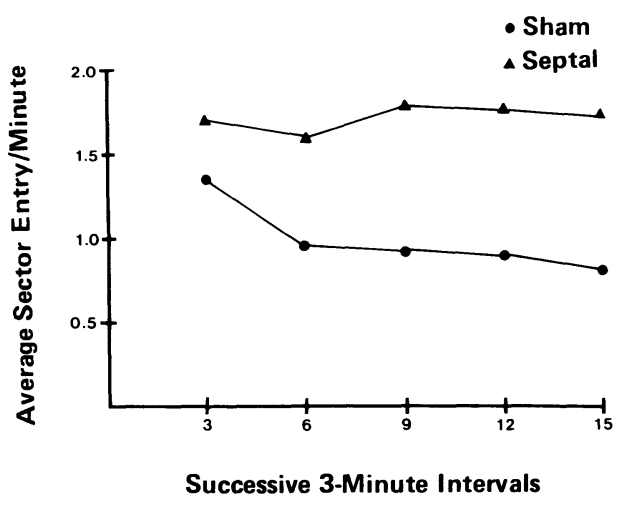

Figure 3. Averaged rate of sector-entry behavior for septals and normal rats in successive 3-min epochs of the daily 15-min exploratory phase.

so obtained, together with the overall Trial 1 performance, was plotted for each animal in Figure 4.

As can be seen from Figure 4, of the 19 animals in the two groups, 10 (8 shams and 2 septals) had habituation indices higher than the average index for the combined groups $(p=.005$, Fisher exact probability test). However, of the 10 animals that did decrease their sector-entry rates during the 15 -min exploratory period each day, only 5 (shams) were able to reach criterion on the Trial 1 performance $(p=$ .025 , Fisher's exact probability test). It is clear from these data that a decrease in rate of sector entry during the exploratory phase of the three-table task does not ensure successful Trial 1 performance.

For shams, there was a decrease in sector entry rate during the 15 -min exploratory period on $70 \%$ of the occasions in which these animals performed correctly on Trial 1. In contrast, septal animals showed a de-

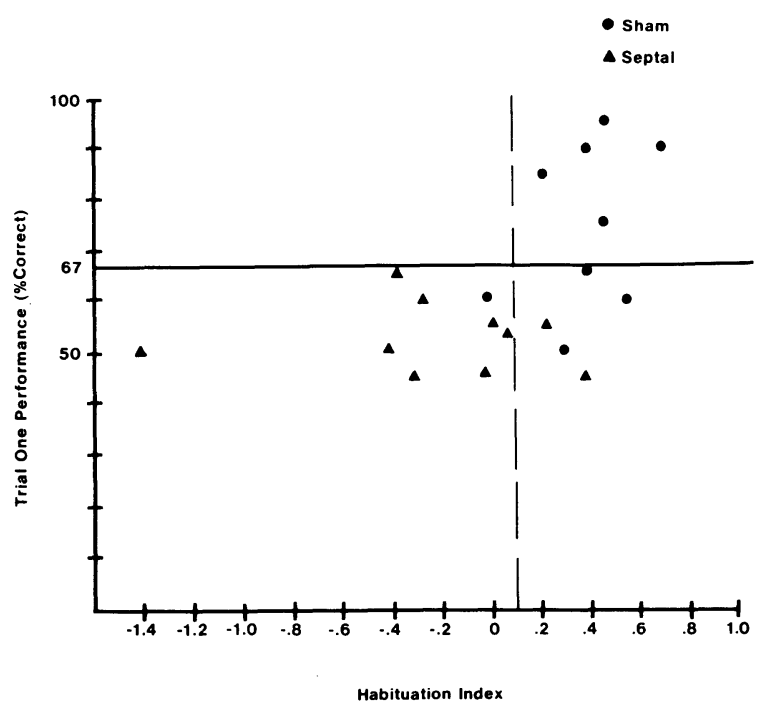

Figure 4. Relation between habituation index $(\mathbf{A}-\mathbf{B}) / \mathrm{A}$, and Trial 1 performance. crease in sector entry rate on $41 \%$ of the occasions in which they were correct on Trial 1 . In addition, only $9 \%$ of the correct responses shown by septal animals on Trial 1 were contrary to the turning preference expressed during the testing period. Thus, septal animals would be correct on Trial 1 when the direction of the correct table happened to correspond to the turn preference. The fact that the shams scored at better than $70 \%$ correct on Trial 1 indicates that a different mechanism underlies their correct performance on Trial 1.

There was no anatomical basis for the difference between the two septal animals' demonstrating a reduction in sector entry rate and those not demonstrating habituation. The characteristics of the lesion subgroupings described above (see histological results) were present in both groups of septal animals.

Finally, it will be recalled that septal animals demonstrated a marked tendency to repeat the same turn on each day on Trial 1 and also to repeat their Trial 1 response on Trials 2 and 3 . Is this perseverative tendency manifested not only in the test trial situation, but also when the animals are merely engaged in locomoting about the environment during the exploratory phase? For this analysis, a perseverative tendency during the exploratory phase was defined as twice as many turns in one direction than in the other during the 15-min exploratory phase. The number of days that such a tendency was observed for each animal was divided by the total number of exploratory phases $(\mathrm{N}=20)$, to yield a measure reflecting the relative number of days in which perseveration was observed. When this value was correlated with the tendency to perseverate on Trial 1 of the 20-day test period, no significant relation was found for septals $\left(r_{s}=.4149, p>.05\right)$ or shams $\left(r_{s}=.406, p>.05\right)$. The perseverative tendency during exploration was also found to be statistically unrelated to the tendency to repeat, on Trials 2 and 3 , the turn response made on the first trial of each day's testing (septals, $r_{s}=.2893, p>.05$; shams, $r_{s}=.0125$, $\mathrm{p}>.05$ ). Thus, it is clear that the perseverative tendency in the test situation does not reflect the operation of the same mechanism controlling perseveration during the exploratory phase.

\section{DISCUSSION}

This study addressed the question of whether the failure of septally damaged rats to perform correctly on the daily first trial of the three-table task was related to a failure to explore during the exploratory phase of the task. This issue derived from the fact that septal animals engage in significantly more sector entries during the 15-min exploratory period than do normal or sham-operated animals (Stahl \& Ellen, 1973, 1979; Figure 2) and the possibility that such an enhancement in sector-entry behavior was merely a 
lesion-induced increase in general activity rather than an increase in exploratory activity per se. The decline in the rate of sector entries during successive 3-min epochs of the 15-min exploratory phase was considered as evidence of exploratory activity ( $\mathrm{O}^{\prime} \mathrm{Keefe}$ $\&$ Nadel, 1978) rather than general activity. The results of the present study (Figure 3) clearly indicated that, on the average, the rate of sector entry behavior for septal animals in the last $3 \mathrm{~min}$ of the 15 -min exploratory period was not reduced below its level in the first $3 \mathrm{~min}$ of the exploratory phase, while that for the shams showed a marked decline. Examination of the performance of individual animals generally confirmed the group data, with only two exceptions in the case of the septals and one in the case of the shams (see Figure 4). These findings would suggest a failure of habituation on the part of the septal animals. This failure to habituate can be interpreted as an absence of exploratory behavior as defined by O'Keefe and Nadel (1978).

Turning now to the question of whether this difference between septals and shams can be used to account for the failure of the septal animals to reach criterion on Trial 1, we found that although 10 animals (8 shams and 2 septals) were able to decrease their rates of sector entry behavior during the exploratory period, only 5 animals (shams) were able to perform at or above criterion on Trial 1 of the three-table task. These findings lead to two general conclusions: first, the decreased rate of sector-entry behavior reflects the acquisition of information during the exploration phase that is necessary for successful performance (Ellen et al., 1982); and second, habituation (i.e., becoming familiar with the problem space) does not ensure successful problem solving on Trial 1. This latter conclusion would imply that although learning the spatial relations among the tables during the exploratory period may be necessary (Stahl \& Ellen, 1974; Herrmann et al., 1982), it is not a sufficient precondition for successful Trial 1 performance. In fact, as indicated earlier, septal animals were correct on Trial 1 when the correct table happened to correspond to the turn preference. Thus, even though two of the septal animals had habituation indices higher than the average of the group as a whole, the turning preference resulting from the septal damage was a more potent determinant of the direction of the Trial 1 response than was the locus of the food table.

There is one final issue that needs to be addressed. It will be recalled that none of the septal animals adopted a spatial solution to the problem (i.e., running directly from the start to the baited table at criterion levels or better on Trial 1). Rather, these animals repeated the same turning response on Trial 1 from day to day. They also tended to repeat, on Trials 2 and 3, the turn made on Trial 1 for that day regardless of the correct or incorrect outcome of the
Trial 1 response (see Table 2). This perseverative tendency was not correlated with the perseverative behavior shown by the septal animals during the exploratory phase of the problem. This fact would suggest that the perseverative tendency seen in the test-trial situation is actually a septally damaged rat's adaptation to the test trial situation itself rather than a simple lesion-induced response bias expressed regardless of whether the animal is in a test trial or merely on an exploratory expedition. Although the perseveration of a turn response is an adaptive response to the test-trial situation, it remains to be determined whether it is a consequence or a cause of the animal's inability to adopt a spatial solution in the test-trial situation.

\section{REFERENCES}

Bolles, R. C. Theory of motivation. New York: Harper \& Row, 1967.

Ellen, P., \& Powell, E. W. Effects of septal lesions on behavior generated by positive reinforcement. Experimental Neurology, 1962, 6, 1-11. (a)

Ellen, P., \& Powell, E. W. Temporal discrimination in rats with rhinencephalic lesions. Experimental Neurology, 1962, 6, 538-547. (b)

Ellen, P., Parko, E. M., Wages, C., Doherty, D., \& HerrmanN, T. Spatial problem solving by rats: Exploration and cognitive maps. Learning and Motivation, 1982, 13, $81-94$.

Herrmann, T., Bahr, E., Bremner, B., \& Ellen, P. Problem solving in the rat: Stay vs. shift solutions on the three-table task. Animal Learning \& Behavior, 1982, 10, 39-45.

Herrmann, T., Black, A. H., Anschel, H., \& Ellen, P. Comparison of septal and fornical lesioned rats performance on the Maier three-table reasoning task. Physiology \& Behavior, 1978, 20, 297-302.

Herrmann, T., Black, A. H., Doherty, D., \& Ellen, P. Visual cues fail to attenuate deficits on a spatial-integration task following septal or fornical damage. Physiological Psychology, 1980, 8, 29-32.

Maie R, N.R.F. The effects of cerebral destruction on reasoning and learning in rats. Journal of Comparative Neurology, 1932, 54, 47-55. (a)

MAIER, N.R.F. Cortical destruction of the posterior part of the brain and its effect on reasoning in rats. Journal of Comparative Neurology, 1932, 56, 179-214. (b)

O'KeEFE, J., \& NADEL, L. The hippocampus as a cognitive map. Oxford: Clarendon Press, 1978.

Pellegrino, L. J., \& Cushman, A. J. A stereotaxic atlas of the rat brain. New York: Meredith, 1967.

RABE, A., \& HADDAD, R. K. Integrative deficit after hippocampal lesions. Proceedings of the 77th Annual Convention of the American Psychological Association, 1969, 2, 213-214.

Stahl, J. M., \& Ellen, P. Septal lesions and reasoning performance in the rat. Journal of Comparative and Physiological Psychology, 1973, 84, 629-638.

Stahl, J. M., \& Ellen, P. Factors in the reasoning performance of the rat. Journal of Comparative and Physiological Psychology, 1974, 87, 598-604.

Stahl, J. M., \& Ellen, P. Performance of rats on the Maier three-table task following septal lesions occurring 24 hours after birth. Journal of Comparative and Physiological Psychology, 1979, 93, 1145-1153.

(Manuscript received December 10, 1982; revision accepted for publication March 28, 1983.) 